\title{
Interactive comment on "Unravelling the physical and physiological basis for the solar-induced chlorophyll fluorescence and photosynthesis relationship" by Peiqi Yang et al.
}

Peiqi Yang et al.

p.yang@utwente.nl

Received and published: 2 November 2020

The comment was uploaded in the form of a supplement:

https://bg.copernicus.org/preprints/bg-2020-323/bg-2020-323-AC3-supplement.pdf

Interactive comment on Biogeosciences Discuss., https://doi.org/10.5194/bg-2020-323, 2020. 